\title{
Moving Frames for Heart Fiber Reconstruction
}

\author{
Emmanuel Piuze $^{a}$, Jon Sporring ${ }^{b}$, and Kaleem Siddiqi ${ }^{a}$ \\ ${ }^{a}$ School of Computer Science \& Centre for Intelligent Machines, McGill University. \\ ${ }^{b}$ eScience Center, Department of Computer Science, University of Copenhagen.
}

\begin{abstract}
The method of moving frames provides powerful geometrical tools for the analysis of smoothly varying frame fields. However, in the face of missing measurements, a reconstruction problem arises, one that is largely unexplored for 3D frame fields. Here we consider the particular example of reconstructing impaired cardiac diffusion magnetic resonance imaging (dMRI) data. We combine moving frame analysis with a diffusion inpainting scheme that incorporates rule-based priors. In contrast to previous reconstruction methods, this new approach uses comprehensive differential descriptors for cardiac fibers, and is able to fully recover their orientation. We demonstrate the superior performance of this approach in terms of error of fit when compared to alternate methods. We anticipate that these tools could find application in clinical settings, where damaged heart tissue needs to be replaced or repaired, and for generating dense fiber volumes in electromechanical modelling of the heart.
\end{abstract}

\section{Introduction}

The method of moving frames (MMF), armed with the machinery of exterior calculus, offers tools for the analysis of smoothly varying frame fields. This approach provides useful geometrical descriptors which generalize the concepts of curvature and torsion for space curves, and the shape operator for surfaces [6]. Various problems can be analyzed from a moving frame perspective, including fluid flows in computational mechanics, equivalence and symmetry problems in physics [4], and invariant geometrical features in computer vision. The latter includes the geometry and evolution of curves in the euclidean, affine and projective planes [5], the differential geometry of motion paths [7] and the classical structure from motion problem [3].

In applications that involve acquired or fitted frame data, regions with missing measurements require data reconstruction. Although there exists a literature on the inpainting of $1 \mathrm{D}$ and $2 \mathrm{D}$ signals [11], the case of 3D frame fields is relatively unexplored. Here we consider the reconstruction of such frame fields fit to cardiac diffusion magnetic resonance imaging (dMRI) data. Prior work has shown that for normal hearts such frames rotate smoothly in the myocardium, lending them to moving frame analysis $[9,12]$. The presence of pathologies can upset this organization and impair the ability of the heart muscle to operate efficiently. Modern treatments of such conditions include tissue engineering, where a synthetic material or stem cell therapy is used to mimic cardiomyocyte growth 
to restore cardiac contractile muscle properties in vitro [14], and mechanical ventricular restoration techniques that involve reconstruction, cauterization, and/or ablation of impaired or necrotic regions. Clinical studies have demonstrated that these techniques effectively improve ventricular function [1]. However, at present no formal rigorous geometrical constraints are used to guide or validate the reconstruction process. Among the presently available techniques for recovering missing information in dMRI are the interpolation of the diffusion tensor or the diffusion signal itself [15] and the application of rule-based methods [2].

Motivated by the recovery of fiber differential signatures using atlas-based methods in [8], we consider a new approach where moving frame analysis is combined with an intrinsic diffusion inpainting scheme that also allows for the inclusion of cardiac image priors. In contrast to [8] where only differential signatures were recovered, this new approach also recovers the local fiber direction, which is critical for most applications such as electromechanical modelling and guidance [2]. We demonstrate the use of our method for reconstruction in test settings simulating prototypical dMRI volumes and show that it achieves low error compared to other alternatives. Our key contributions include the development of a novel closed-form method for connection forms in 3D frame fields, and its use in a frame field inpainting scheme.

The paper is organized as follows. In Sec. 2 we review basic principles of cardiac fiber anatomy. In Sec. 3 we derive differential descriptors for threedimensional frame fields, following which we present methods for computing them in Sec. 4 and a frame field inpainting scheme in Sec. 5. These tools are then adapted to reconstructing frame fields derived from cardiac dMRI data in Sec. 6. We conclude and review our main contributions in Sec. 7.

\section{Cardiac Fiber Anatomy}

To situate our work we provide a brief review of cardiac anatomy based on [13]. The heart is a hollow, fibromuscular organ with a truncated ellipsoidal shape. Its orientation is determined by a well-defined lower extremity, the apex, and an ill-defined upper part, the base. The heart can be divided in four distinct chambers: the left (LV) and right (RV) ventricles, a pair of synchronized valved muscular pumps, respectively connect with the left (LA) and the right (RA) atria. Structurally, the LV is considerably larger and thicker than the RV, since it pumps oxygenated blood to the entire body. The bulk of the heart muscle is called myocardium. It is principally composed of elongated muscle cells called cardiomyocytes which measure approximately 10-20 $\mu \mathrm{m}$ in diameter and $50-100 \mu \mathrm{m}$ in length. Their primary function is to produce mechanical tension during ejection, but certain specialized cells also serve to conduct electrical activation of the heart muscle. Cardiomyocytes are densely and smoothly packed within a three-dimensional extracellular matrix principally made of connective tissue. The term myofiber is often used as a proxy for localized parallel groups of cardiomyocytes, although they do not exist at a microscopic level. Histological and medical imaging studies have established certain key geometrical proper- 
ties of cardiac myofibers: 1) they form a smoothly varying medium which wraps around each ventricle, 2) this wrapping generates the truncated ellipsoidal shape of the myocardium, 3) focusing on the LV, the helix angle, which is the angle of cardiomyocyte orientation taken with respect to the short-axis plane smoothly rotates from outer to inner wall by a total amount of approximately 120 degrees.

\section{Moving Frames in $\mathbf{R}^{3}$}

Let a point $\boldsymbol{x}=\sum_{i} x_{i} \boldsymbol{e}_{i} \in \mathbf{R}^{3}$ be expressed in terms of $\boldsymbol{e}_{1}, \boldsymbol{e}_{2}, \boldsymbol{e}_{3}$, the natural basis for $\mathbf{R}^{3}$. We define a right-handed orthonormal frame field $\boldsymbol{f}_{1}, \boldsymbol{f}_{2}, \boldsymbol{f}_{3}: \mathbf{R}^{3} \rightarrow$ $\mathbf{R}^{3}$. Each frame axis can be expressed by the rigid rotation $\boldsymbol{f}_{i}=\sum_{j} a_{i j} \boldsymbol{e}_{j}$, where $\boldsymbol{A}=\left\{a_{i j}\right\} \in \mathbf{R}^{3 \times 3}$ is a differentiable attitude matrix such that $\boldsymbol{A}^{-1}=\boldsymbol{A}^{T}$. Treating $\boldsymbol{f}_{i}$ and $\boldsymbol{e}_{j}$ as symbols, we can write [6]

$$
\left[\begin{array}{lll}
\boldsymbol{f}_{1} & \boldsymbol{f}_{2} & \boldsymbol{f}_{3}
\end{array}\right]^{T}=\boldsymbol{A}\left[\begin{array}{lll}
\boldsymbol{e}_{1} & \boldsymbol{e}_{2} & \boldsymbol{e}_{3}
\end{array}\right]^{T} .
$$

Since each $\boldsymbol{e}_{i}$ is constant, the differential geometry of the frame field is completely characterized by $\boldsymbol{A}$. Taking the exterior derivative on both sides, we have

$$
\mathrm{d}\left[\begin{array}{lll}
\boldsymbol{f}_{1} & \boldsymbol{f}_{2} & \boldsymbol{f}_{3}
\end{array}\right]^{T}=(\mathrm{d} \boldsymbol{A}) \boldsymbol{A}^{-1}\left[\begin{array}{lll}
\boldsymbol{f}_{1} & \boldsymbol{f}_{2} & \boldsymbol{f}_{3}
\end{array}\right]^{T}=\boldsymbol{C}\left[\begin{array}{lll}
\boldsymbol{f}_{1} & \boldsymbol{f}_{2} & \boldsymbol{f}_{3}
\end{array}\right]^{T},
$$

where d denotes the exterior derivative, and $\boldsymbol{C}=(\mathrm{d} \boldsymbol{A}) \boldsymbol{A}^{-1}=\left\{c_{i j}\right\} \in \mathbf{R}^{3 \times 3}$ is the Maurer-Cartan matrix of connection forms $c_{i j}$. Writing $\boldsymbol{f}_{i}$ as symbols, (2) is to be understood as $\mathrm{d} \boldsymbol{f}_{i}=\sum_{j} c_{i j} \boldsymbol{f}_{j}$. The Maurer-Cartan matrix is skew symmetric [6], hence we have

$$
\boldsymbol{C}=\left[\begin{array}{ccc}
0 & c_{12} & c_{13} \\
-c_{12} & 0 & c_{23} \\
-c_{13} & -c_{23} & 0
\end{array}\right],
$$

such that there are at most 3 independent, non-zero 1-forms: $c_{12}, c_{13}$, and $c_{23}$. 1forms operate on tangent vectors through a process denoted contraction, written as $\mathrm{d} w\langle\boldsymbol{v}\rangle \in \mathbf{R}$ for a general 1-form $\mathrm{d} w=\sum_{i} w_{i} \mathrm{~d} \boldsymbol{e}_{i}$ and tangent vector $\boldsymbol{v}$ on $\mathbf{R}^{3}$, which yields $\mathrm{d} w\langle\boldsymbol{v}\rangle=\sum_{i} w_{i} \mathrm{~d} \boldsymbol{e}_{i}\left\langle\sum_{j} v_{j} \boldsymbol{e}_{j}\right\rangle=\sum_{i} w_{i} v_{i}$, since d $\boldsymbol{e}_{i}\left\langle\boldsymbol{e}_{j}\right\rangle=\delta_{i j}$, where $\delta_{i j}$ is the Kronecker delta.

The space of linear models for smooth frame fields is fully parametrized by the 1 -forms $c_{i j}$. This space can be explored by considering the motion of $\boldsymbol{f}_{i}$ in a direction $\boldsymbol{v}=\sum_{k} v_{k} \boldsymbol{f}_{k}$, using the first order terms of a Taylor series centered at $\boldsymbol{x}_{0}$ :

$$
\tilde{\boldsymbol{f}}_{i}\left(\boldsymbol{x}_{0}+\boldsymbol{v}\right)=\boldsymbol{f}_{i}+\mathrm{d} \boldsymbol{f}_{i}\langle\boldsymbol{v}\rangle+\mathcal{O}\left(\|\boldsymbol{v}\|^{2}\right) \approx \boldsymbol{f}_{i}+\sum_{j \neq i} \boldsymbol{f}_{j} \sum_{k} v_{k} c_{i j k},
$$

where $\boldsymbol{f}_{i}$ and $\mathrm{d} \boldsymbol{f}_{i}$ are evaluated at $\boldsymbol{x}_{0}$, and $c_{i j k} \equiv c_{i j}\left\langle\boldsymbol{f}_{k}\right\rangle$ are the connection forms of the local frame. Since only 3 unique non-zero combinations of $c_{i j}$ are possible, there are in total 9 connections $c_{i j k}$. These coefficients express the rate 
of turn of the frame vector $\boldsymbol{f}_{i}$ towards $\boldsymbol{f}_{j}$ when $\boldsymbol{x}$ moves in the direction $\boldsymbol{f}_{k}$. Fig. 1 illustrates the behavior of the frame field described by $c_{i j k}$. For example, with $\boldsymbol{f}_{1}$ taken to be the local orientation of a fiber and $\boldsymbol{f}_{3}$ taken to be the component of the heart wall normal orthogonal to $\boldsymbol{f}_{1}, c_{131}$ measures the circumferential curvature of a fiber and $c_{123}$ measures the change in its helix angle [9].
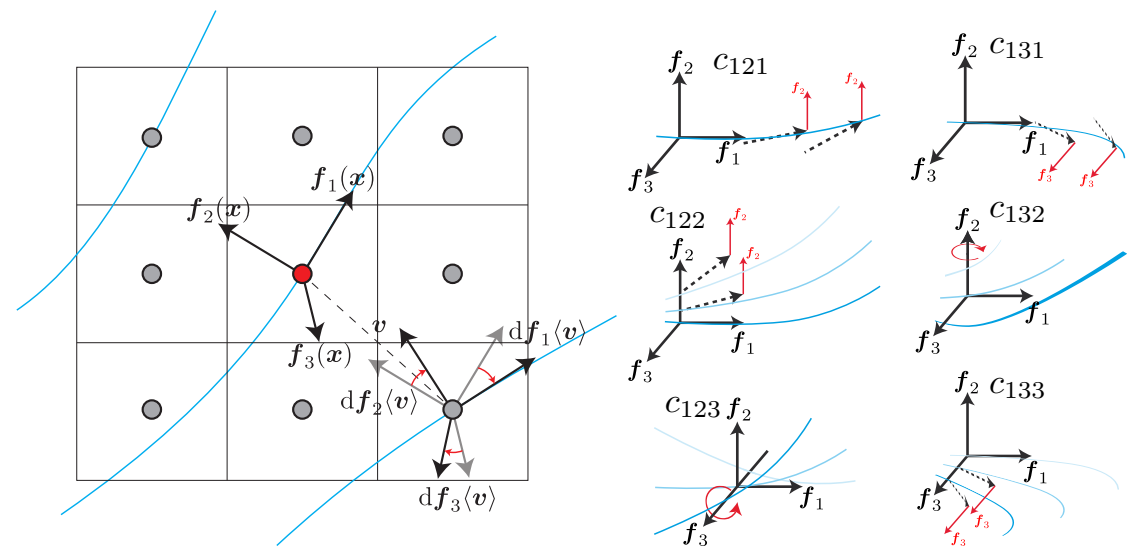

Fig. 1: (Left) Turning of frame axes at $\boldsymbol{x}$ expressed in the local basis $\boldsymbol{f}_{1}, \boldsymbol{f}_{2}, \boldsymbol{f}_{3}$ when $\boldsymbol{x}$ moves in the direction $\boldsymbol{v}$. (Right) frame field variation characterized by the connections $c_{i j k}$ for $i=1$ ( $c_{23 k}$ are not shown).

\section{Computation of Connection Forms}

A first order generator for frame fields using (4) requires knowledge of the underlying connection forms $c_{i j k}$. We shall explore three ways of computing these: 1) a direct estimate based on finite differences, 2) a regularized optimization scheme, and 3) a novel closed-form computation which yields exact results on linear manifolds. In Sec. 4.4 we discuss conditions under which each method could be used, and later in Sec. 5 we use various combinations of these for inpainting 3D frame fields.

\subsection{Connections via Finite Differentiation}

In smooth frame fields, the connection 1-forms $c_{i j}$ can be directly obtained using (2), i.e., $\mathrm{d} \boldsymbol{f}_{i} \cdot \boldsymbol{f}_{k}=\left(\sum_{j}^{3} c_{i j} \boldsymbol{f}_{j}\right) \cdot \boldsymbol{f}_{k}=\sum_{j}^{3} c_{i j} \delta_{j k}=c_{i k}$. The differentials d $\boldsymbol{f}_{i}$ can be computed by applying the exterior derivative for a function, i.e., for the $k^{\prime}$ th component of $\boldsymbol{f}_{i}, \boldsymbol{f}_{i k}: \mathbf{R}^{3} \rightarrow \mathbf{R}, \mathrm{d} \boldsymbol{f}_{i k}=\sum_{l}^{3} \frac{\partial \boldsymbol{f}_{i k}}{\partial x_{l}} \mathrm{~d} \boldsymbol{e}_{l}$,

$$
\mathrm{d} \boldsymbol{f}_{i} \cdot \boldsymbol{f}_{j}\langle\boldsymbol{v}\rangle=\boldsymbol{f}_{j}^{T} \mathrm{~d} \boldsymbol{f}_{i}\langle\boldsymbol{v}\rangle=\sum_{k}^{3} \sum_{l}^{3} \boldsymbol{f}_{j k} \frac{\partial \boldsymbol{f}_{i k}}{\partial x_{l}} \mathrm{~d} \boldsymbol{e}_{l}\langle\boldsymbol{v}\rangle=\boldsymbol{f}_{j}^{T} \mathbf{J}_{i} \boldsymbol{v}
$$


where $\mathbf{J}_{i}=\left[\frac{\partial \boldsymbol{f}_{i p}}{\partial x_{q}}\right] \in \mathbf{R}^{3 \times 3}$ is the Jacobian matrix of partial derivatives of $\boldsymbol{f}_{i}$. Setting $\boldsymbol{v}=\boldsymbol{f}_{k}$, we obtain

$$
c_{i j k}=\boldsymbol{f}_{j}^{T} \mathbf{J}_{i} \boldsymbol{f}_{k} .
$$

The Jacobian matrix $\mathbf{J}_{i}$ can be approximated to first order using, e.g., finite differences on $\boldsymbol{f}_{i}$ with a spacing of size $\delta x: \frac{\partial \boldsymbol{f}_{i j}}{\partial x_{k}}(\boldsymbol{x}) \approx \frac{\boldsymbol{f}_{i j}\left(\boldsymbol{x}+\boldsymbol{e}_{k}\right)-\boldsymbol{f}_{i j}(\boldsymbol{x})}{\delta x}$.

\subsection{Connections via Energy Minimization}

The connection forms $c_{i j k}$ at a point $\boldsymbol{x}_{0}$ can also be obtained as the minimizer of an extrapolation energy $\mathcal{E}$ contained within a neighborhood $\Omega$ :

$$
c_{i j k}^{*}\left(\boldsymbol{x}_{0}\right)=\arg \min _{c_{i j k}} \mathcal{E}\left(\boldsymbol{x}_{0}, \Omega\right)+\lambda\left|c_{i j k}\right|,
$$

where $\lambda$ is a regularization weight used to penalize high curvature. Denoting $\tilde{\boldsymbol{f}}_{i}$ as the normalized approximation to $\boldsymbol{f}_{i}$ at $\boldsymbol{x}_{0}+\boldsymbol{v}$ using (4), we follow [8] and choose $\mathcal{E}$ to minimize the angular error between $\tilde{\boldsymbol{f}}_{i}$ and $\boldsymbol{f}_{i}: \mathcal{E}\left(\boldsymbol{x}_{0}, \Omega\right)=$ $\frac{1}{|\Omega|} \sum_{\boldsymbol{v} \in \Omega} \sum_{i}^{3} \varepsilon_{i}\left(\boldsymbol{x}_{0}+\boldsymbol{v}\right)$, with $\varepsilon_{i}\left(\boldsymbol{x}_{0}+\boldsymbol{v}\right)=\arccos \left(\boldsymbol{f}_{i}\left(\boldsymbol{x}_{0}+\boldsymbol{v}\right) \cdot \tilde{\boldsymbol{f}}_{i}\left(\boldsymbol{x}_{0}+\boldsymbol{v}\right)\right)$.

\subsection{Closed-Form Connections in Linear Space}

A disadvantage of using the previous energy minimization approach is that coupling between the connections $c_{i j k}$ is not explicitly enforced, i.e., the requirement that $c_{i j}\langle\boldsymbol{v}\rangle=\sum_{k} c_{i j k} v_{k}$. Thus it may lead to non-integrable differential descriptors. We now develop a novel way of computing connection forms that is based on trigonometrical considerations in the first-order structure of 3D frame fields and which enforces that coupling. This method also provides exact $c_{i j k}$ measurements in manifolds that have low second-order curvatures $\left(\mathrm{d}^{2} \boldsymbol{f}_{i} \rightarrow 0\right)$. Given a local basis $\boldsymbol{f}_{i}$ and data-driven neighboring bases $\boldsymbol{f}_{i}(\boldsymbol{v} \in \Omega)$, the 1-forms $c_{i j}\langle\boldsymbol{v}\rangle$ can be solved for using linear least-squares. We begin by expanding (4),

$$
\boldsymbol{f}_{i}(\boldsymbol{v})=\boldsymbol{f}_{i}+c_{i j}\langle\boldsymbol{v}\rangle \boldsymbol{f}_{j}+c_{i k}\langle\boldsymbol{v}\rangle \boldsymbol{f}_{k}
$$

and analyze this expression geometrically using Fig. 2. Let $\boldsymbol{f}_{i}^{j}(\boldsymbol{v})$ denote the projection of $\boldsymbol{f}_{i}(\boldsymbol{v})$ in the $\boldsymbol{f}_{i}-\boldsymbol{f}_{j}$ plane, i.e., $\boldsymbol{f}_{i}^{j}(\boldsymbol{v})=\boldsymbol{f}_{i}(\boldsymbol{v})-\left(\boldsymbol{f}_{i}(\boldsymbol{v}) \cdot \boldsymbol{f}_{k}\right) \boldsymbol{f}_{k}, \quad k \in$ $(1,2,3) \neq i \neq j$, and let $\theta_{i j}(\boldsymbol{v})$ denote the signed angle between $\boldsymbol{f}_{i}$ and $\boldsymbol{f}_{i}^{j}(\boldsymbol{v})$ with positive values assigned to $\theta_{i j}(\boldsymbol{v})$ rotating $\boldsymbol{f}_{i}$ towards $\boldsymbol{f}_{j}$, obtained as

$$
\theta_{i j}(\boldsymbol{v})=\operatorname{sgn}\left(\boldsymbol{f}_{i}^{j}(\boldsymbol{v}) \cdot \boldsymbol{f}_{j}\right) \cdot \arccos \left(\left|\boldsymbol{f}_{i} \cdot \boldsymbol{f}_{i}^{j}(\boldsymbol{v}) /\left\|\boldsymbol{f}_{i}^{j}(\boldsymbol{v})\right\|\right|\right) .
$$

Using trigonometry, we obtain

$$
\tan \left(\theta_{i j}(\boldsymbol{v})\right)=\frac{c_{i j}\langle\boldsymbol{v}\rangle}{\left\|\boldsymbol{f}_{i}\right\|}=c_{i j}\langle\boldsymbol{v}\rangle \quad \text { since } \boldsymbol{f}_{i} \text { is a unit vector. }
$$


Expanding the contraction $c_{i j}\langle\boldsymbol{v}\rangle$ :

$$
\begin{aligned}
c_{i j}\langle\boldsymbol{v}\rangle & =\left(\boldsymbol{v} \cdot \boldsymbol{f}_{1}\right) c_{i j 1}+\left(\boldsymbol{v} \cdot \boldsymbol{f}_{2}\right) c_{i j 2}+\left(\boldsymbol{v} \cdot \boldsymbol{f}_{3}\right) c_{i j 3} \\
& =v_{1} c_{i j 1}+v_{2} c_{i j 2}+v_{3} c_{i j 3} \quad \text { with } v_{k} \equiv \boldsymbol{v} \cdot \boldsymbol{f}_{k} \\
& \Rightarrow\left[c_{12}\langle\boldsymbol{v}\rangle c_{13}\langle\boldsymbol{v}\rangle c_{23}\langle\boldsymbol{v}\rangle\right]=\left[\begin{array}{lll}
v_{1} & v_{2} & v_{3}
\end{array}\right]\left[\begin{array}{lll}
c_{121} & c_{131} & c_{231} \\
c_{122} & c_{132} & c_{232} \\
c_{123} & c_{133} & c_{233}
\end{array}\right]
\end{aligned}
$$

and substituting in (10), we get a linear system in the 9 unknowns $c_{i j k}$ :

$$
\left[\begin{array}{l}
v_{1} \\
v_{2} \\
v_{3}
\end{array}\right]^{T}\left[\begin{array}{lll}
c_{121} & c_{131} & c_{231} \\
c_{122} & c_{132} & c_{232} \\
c_{123} & c_{133} & c_{233}
\end{array}\right]=\left[\begin{array}{l}
\tan \left(\theta_{12}(\boldsymbol{v})\right) \\
\tan \left(\theta_{13}(\boldsymbol{v})\right) \\
\tan \left(\theta_{23}(\boldsymbol{v})\right)
\end{array}\right]^{T}
$$

Aggregating $n$ measurements $\boldsymbol{v}_{i} \in \Omega(\boldsymbol{x})$, we have

$$
\underbrace{\left[\begin{array}{ccc}
v_{11} & v_{12} & v_{13} \\
& \vdots \\
v_{i 1} & v_{i 2} & v_{i 3} \\
\vdots & \\
v_{n 1} & v_{n 2} & v_{n 3}
\end{array}\right]}_{\boldsymbol{V} \in \mathbf{R}^{n \times 3}} \underbrace{\left[\begin{array}{ccc}
c_{121} & c_{131} & c_{231} \\
c_{122} & c_{132} & c_{232} \\
c_{123} & c_{133} & c_{233}
\end{array}\right]}_{\boldsymbol{C} \in \mathbf{R}^{3 \times 3}}=\underbrace{\left[\begin{array}{c}
\tan \left(\theta_{12}\left(\boldsymbol{v}_{1}\right)\right) \tan \left(\theta_{13}\left(\boldsymbol{v}_{1}\right)\right) \tan \left(\theta_{23}\left(\boldsymbol{v}_{1}\right)\right) \\
\vdots \\
\tan \left(\theta_{12}\left(\boldsymbol{v}_{i}\right)\right) \tan \left(\theta_{13}\left(\boldsymbol{v}_{i}\right)\right) \tan \left(\theta_{23}\left(\boldsymbol{v}_{i}\right)\right) \\
\vdots \\
\tan \left(\theta_{12}\left(\boldsymbol{v}_{n}\right)\right) \tan \left(\theta_{13}\left(\boldsymbol{v}_{n}\right)\right) \tan \left(\theta_{23}\left(\boldsymbol{v}_{n}\right)\right)
\end{array}\right]}_{\tilde{C} \in \mathbf{R}^{n \times 3}}
$$

We need $n \geq 3$ otherwise the system will be undetermined. In general, $\boldsymbol{v}_{i} \cdot \boldsymbol{v}_{j} \neq 0$ such that $\boldsymbol{V}$ is not full row rank. A QR factorization or singular value decomposition (SVD) pseudoinverse can be used to solve for $\boldsymbol{C}=\left(\boldsymbol{V}^{T} \boldsymbol{V}\right)^{-1} \boldsymbol{V}^{T} \tilde{\boldsymbol{C}}$.

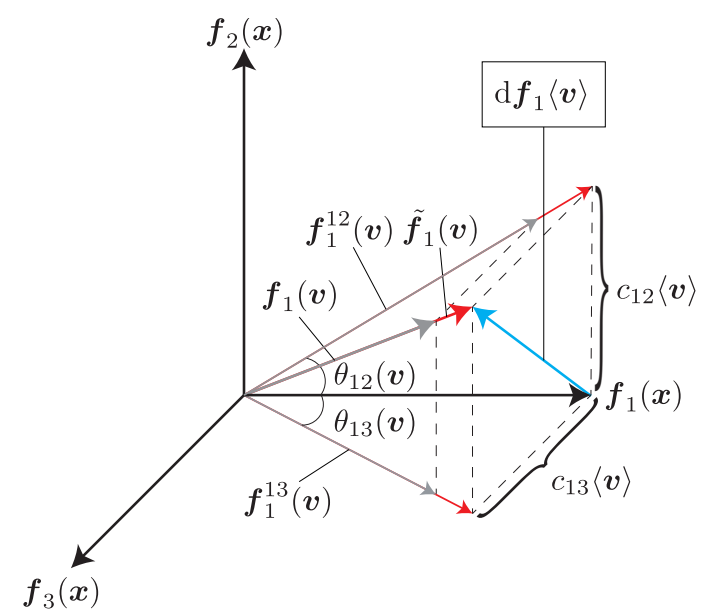

Fig. 2: Differential of the frame axis $\boldsymbol{f}_{1}(\boldsymbol{x})$ expressed in local coordinates. 


\subsection{Method Comparison}

We compare the three previous methods by computing mean connection forms along with fitting errors in a neighborhood containing 50K voxels in Fig. 3. The frame field data is from a healthy rat heart, taken from [12], where $\boldsymbol{f}_{1}$ is the fiber direction, $\boldsymbol{f}_{3}$ is approximately normal to the heart wall, and $\boldsymbol{f}_{2}=$ $\boldsymbol{f}_{3} \times \boldsymbol{f}_{1}$. For the energy-minimizing approach we experimented with NelderMead (NM) and BOBYQA (BQ) [10] optimizers $^{1}$ to solve the energy term in (7). Here, all connection forms are estimated in an isotropic neighborhood of size $3^{3}$, seeds are obtained using (6), and $\lambda=0.0001$. On an SSD quad-core $2.5 \mathrm{GHz}$ machine, fitting $1 \mathrm{k}$ voxels takes about $3 \mathrm{~ms}$ using finite differentiation, $100 \mathrm{~ms}$ using 200 Nelder-Mead iterations, 30ms using closed-form estimation, and 1s using BOBYQA optimization. Seeded NM computations converge faster than unseeded, and reach comparable values at about 150 iterations. BOBYQA yields a slightly lower error but is impractical due its large time complexity. Our closedform method yields a slightly larger global error than both optimizers beyond 100 iterations but has the advantage of being considerably faster, and, unlike the other methods, offers the theoretical advantage of enforcing integrability of the reconstructed frame field while being exact to first order.
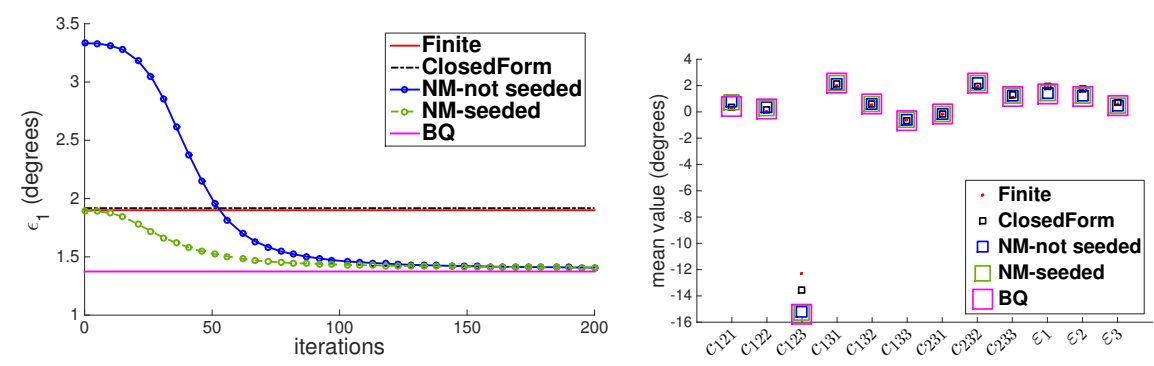

Fig. 3: (Left) Mean $\boldsymbol{f}_{1}$ fitting error $\epsilon_{1}$ (radians), and (right) mean connection forms and errors (radians) for different $c_{i j k}$ estimation techniques in 50k voxels.

\section{Frame Field Reconstruction}

We now describe our frame field inpainting procedure. Here, $\Omega$ is a region where frame field data is available, and $A$ is a reconstruction domain. Starting with $A^{0}=A$, each iteration propagates information on the boundary $\partial A^{n}$ such that $\partial A^{n+1}=\partial A^{n} \oplus B_{r}$ is eroded with a ball element $B_{r}$ of radius $r=1$ and $c_{i j k}$ is updated in $\Omega$ and $\partial A^{n+1}$ until $A^{n+1}=\emptyset$. Frames $\boldsymbol{f}_{i}$ are transported from $\boldsymbol{x} \in \partial A^{n}$ in a direction $\boldsymbol{v}$ across $\partial A$ using a neighbor accumulation of (4),

$$
\left.\tilde{\boldsymbol{f}}_{i}^{n+1}\right|_{\boldsymbol{x}+\boldsymbol{v}}=\sum_{\boldsymbol{x} \in \partial A^{n}}\left(\left.\boldsymbol{f}_{i}^{n}\right|_{\boldsymbol{x}}+\left.\left.\sum_{j \neq i} \boldsymbol{f}_{j}^{n}\right|_{\boldsymbol{x}} \sum_{k} c_{i j k}(\boldsymbol{x}+\boldsymbol{v}) \cdot \boldsymbol{f}_{k}^{n}\right|_{\boldsymbol{x}}\right),
$$

\footnotetext{
${ }^{1}$ See [10] for these and other optimization strategies.
} 
which is then normalized. Connection forms $c_{i j k}$ at $\boldsymbol{x} \in \partial A^{n}$ are obtained by combining the three methods described in Sec. 4, based on the following heuristics $^{2}$, where $\kappa=\left\|\boldsymbol{V}^{-1}\right\|_{F}\|\boldsymbol{V}\|_{F}$ is the condition number of $\boldsymbol{V}$ in (15) and $F$ is the Frobenius norm:

$$
c_{i j k}(\boldsymbol{x})=\left\{\begin{array}{l}
\text { Closed-form of }(15): \boldsymbol{x} \text { has }>N \text { neighbors, and } \kappa<\kappa_{0} \\
\text { Energy of (7) with } \lambda=0.0001 \text { and seeding with }(6): \text { else, }
\end{array}\right.
$$

where $N=\frac{1}{2}(2 r+1)^{3}$ and $\kappa_{0}=3$ were determined empirically and generally offer a good tradeoff between neighborhood connectivity and well-conditioning of $\boldsymbol{V}$. Unrealistically large $c_{i j k}$ values can still arise in spite of the regularization. To see this, take $\boldsymbol{f}_{1}(\boldsymbol{x})$ and make it parallel to $\boldsymbol{f}_{2}(\boldsymbol{x})$ in a neighboring voxel. Using (4), we have $\tilde{\boldsymbol{f}}_{1}(\boldsymbol{v}) \approx \boldsymbol{f}_{1}+c_{12}\langle\boldsymbol{v}\rangle \boldsymbol{f}_{2}+c_{13}\langle\boldsymbol{v}\rangle \boldsymbol{f}_{3}$, such that $\boldsymbol{f}_{1}(\boldsymbol{v}) \backslash \backslash \boldsymbol{f}_{2} \Rightarrow c_{12}\langle\boldsymbol{v}\rangle \rightarrow$ $\infty$. When necessary, we thus apply a hard threshold on $\mathcal{E}$ and $c_{i j k}$ in (17). We set $c_{i j k}=0$ if $\mathcal{E}>\frac{\pi}{4}$, or if $c_{i j k}$ exceeds bounds obtained as follows. In the discrete case using forward differences, the frame axis differential d $\boldsymbol{f}_{i}=\mathrm{d}\left(\boldsymbol{f}_{i 1} \boldsymbol{e}_{1}+\boldsymbol{f}_{i 2} \boldsymbol{e}_{2}+\right.$ $\left.\boldsymbol{f}_{i 3} \boldsymbol{e}_{3}\right)$ is bounded since $\left|\frac{\partial \boldsymbol{f}_{i}}{\partial x_{k}}\right| \approx \frac{1}{2}\left|\boldsymbol{f}_{i j}\left(\boldsymbol{x}+\boldsymbol{e}_{k}\right)-\boldsymbol{f}_{i j}(\boldsymbol{x})\right| \leq 1$ and $\left\|\boldsymbol{f}_{i}\right\|=1$. We thus have $\left|c_{i j}\langle\boldsymbol{v}\rangle\right|=\left|\boldsymbol{f}_{j}^{T} \mathcal{J}_{\boldsymbol{f}_{i}}\left(x_{1}, x_{2}, x_{3}\right) \boldsymbol{v}\right| \leq \boldsymbol{f}_{j}^{T} \cdot\left[\|\boldsymbol{v}\|_{1}\|\boldsymbol{v}\|_{1}\|\boldsymbol{v}\|_{1}\right]^{T} \leq\|\boldsymbol{v}\|_{1}$, and we set $c_{i j k}=\min \left(c_{i j k},\|\boldsymbol{v}\|_{1}\right)$.

The diffusion process guided by (16) and (17) does not enforce orthogonality of the resulting frame field. Since this is a first-order method we expect to see some orthogonality drift as we get deeper into the region $A$. To see this, using (8), we get $\boldsymbol{f}_{1}(\boldsymbol{v}) \cdot \boldsymbol{f}_{2}(\boldsymbol{v})=\left(\boldsymbol{f}_{1}+c_{12}\langle\boldsymbol{v}\rangle \boldsymbol{f}_{2}+c_{13}\langle\boldsymbol{v}\rangle \boldsymbol{f}_{3}\right) \cdot\left(\boldsymbol{f}_{2}+c_{21}\langle\boldsymbol{v}\rangle \boldsymbol{f}_{1}+c_{23}\langle\boldsymbol{v}\rangle \boldsymbol{f}_{3}\right)$ and similarly for the other axis products. Since $\boldsymbol{f}_{i}$ is by definition orthogonal at 0, we have $\boldsymbol{f}_{i} \cdot \boldsymbol{f}_{j}=\delta_{i j}$ such that $\boldsymbol{f}_{i}(\boldsymbol{v}) \cdot \boldsymbol{f}_{j}(\boldsymbol{v})=\left\{\begin{array}{l}1+c_{i j}^{2}+c_{i k}^{2}: i=j \\ c_{i k} c_{j k}: i \neq j\end{array}\right.$ for $k \neq$ $i, k \neq j$. The extrapolated frame $\boldsymbol{f}_{i}(\boldsymbol{v})$ will therefore never be exactly orthonormal. To enforce orthonormality we therefore fix $\boldsymbol{f}_{1}(\boldsymbol{v})$ and find its orthogonal complement $\boldsymbol{f}_{2}^{\perp}(\boldsymbol{v})$ using

$$
\begin{aligned}
\boldsymbol{f}_{2}^{\perp}(\boldsymbol{v}) & =\boldsymbol{f}_{2}-\left(\boldsymbol{f}_{1}(\boldsymbol{v}) \cdot \boldsymbol{f}_{2}(\boldsymbol{v})\right) \boldsymbol{f}_{1}(\boldsymbol{v}) \\
& =\left(-c_{12}-c_{13} c_{23}\right) \boldsymbol{f}_{1}+\left(1-c_{13} c_{23} c_{12}\right) \boldsymbol{f}_{2}+\left(c_{23}-c_{13}^{2} c_{23}\right) \boldsymbol{f}_{3},
\end{aligned}
$$

where $c_{i j}$ is taken as $c_{i j}\langle\boldsymbol{v}\rangle$. We proceed similarly for $\boldsymbol{f}_{3}^{\perp}(\boldsymbol{v})$.

\section{Application to Cardiac Fiber Reconstruction}

Given a partial volume $\Omega$ of fiber orientations $\boldsymbol{f}_{1}$ in a mask $H$ of the heart, we now explore the problem of reconstructing $\boldsymbol{f}_{1}$ everywhere in $A=H-\Omega$.

\subsection{Rule-Based Orientation Priors}

Our inpainting procedure is guided by rule-based priors for fiber orientations based on $H$ and estimated heart wall normals: one relating to the circumferential arrangement of myofibers and the other to their helix angle turning.

\footnotetext{
${ }^{2}$ When Jacobian matrix computations are available using a combination of backward, central, and forward differences, we seed (7) using (6) which improves convergence.
} 
Estimating the circumferential component Using a smoothing kernel $G_{\sigma}$, the Euclidean distance transforms $G_{\sigma} * D_{+}$and $G_{\sigma} * D_{-}$to the outer and inner walls are first computed. From the average $D=\frac{1}{2}\left(D_{+}-D_{-}\right)$local wall normal directions are computed using $\hat{\boldsymbol{f}}_{3}=\nabla D$. The apex $\xi_{0}$ and an upward unit direction as $\hat{\boldsymbol{u}}$ are identified, and used to obtain heart centerline measurements $\xi_{t}$ parametrized over $t$ steps along $\hat{\boldsymbol{u}}, \xi_{t}=\frac{\sum_{x} w(\boldsymbol{x}) \Xi(\boldsymbol{x}) \boldsymbol{x}}{\sum_{\boldsymbol{x}} w(\boldsymbol{x}) \Xi(\boldsymbol{x})}$, where $\Xi(\boldsymbol{x})=\operatorname{sgn}\left(\left|\left(\boldsymbol{x}-\xi_{0}-t \hat{\boldsymbol{u}}\right) \cdot \hat{\boldsymbol{u}}\right|\right)$, i.e., $\Xi(\boldsymbol{x})$ is 1 in the current short axis plane and 0 elsewhere, and $w(\boldsymbol{x})$ is 1 if $\boldsymbol{x}$ is in the myocardium and is 0 otherwise. A smooth heart centerline is then obtained as $L(t)=G_{\sigma} * \xi_{t}$. We can now obtain a local long-axis direction $\boldsymbol{f}_{L}$ using $\boldsymbol{f}_{L}=\frac{\partial L(t)}{\partial t}$ and finally estimate the circumferential direction $\boldsymbol{f}_{c}$ from the cross product of $\boldsymbol{f}_{L}$ and the local wall normal $\hat{\boldsymbol{f}}_{3}$ as $\boldsymbol{f}_{c}=\boldsymbol{f}_{L} \times \boldsymbol{f}_{3}$.

Estimating the helical component We use a rule-based helix angle variation prior from $\alpha^{+}$to $\alpha^{-}$from outer to inner wall, similarly to the work of [2]. A voxel $\boldsymbol{x}$ is first parametrized over the local depth of the heart wall in the range [0,1], where 0 indicates that the voxel is lying on the outer wall and 1 on the inner wall, using $\gamma(\boldsymbol{x})=\frac{D_{+}}{D_{+}+D_{-}}(\boldsymbol{x})=\left(1+D_{-} D_{+}^{-1}\right)^{-1}(\boldsymbol{x}) \in[0,1]$. Then, the local helix angle at $\boldsymbol{x}$ is linearly interpolated using $\alpha(\boldsymbol{x})=\left(\alpha_{+}-\gamma \alpha_{+}+\gamma \alpha_{-}\right)(\boldsymbol{x})$. Finally, the helix fiber direction $\hat{\boldsymbol{f}}_{1}$ is obtained using a helical rotation of $\boldsymbol{f}_{c}$ about the local transmural axis $\hat{\boldsymbol{f}}_{3}$ from the axis angle $\left\langle\hat{\boldsymbol{f}}_{3}, \alpha\right\rangle$ using Rodrigues' formula, $\hat{\boldsymbol{f}}_{1}=\cos \alpha \boldsymbol{f}_{c}+\sin \alpha\left(\hat{\boldsymbol{f}}_{3} \times \boldsymbol{f}_{c}\right)+(1-\cos \alpha)\left(\hat{\boldsymbol{f}}_{3} \cdot \boldsymbol{f}_{c}\right) \hat{\boldsymbol{f}}_{3}$.

Coupling Rule-based Prior and Diffusion Each diffusion pass $n+1$ combines current frame field estimates $\tilde{\boldsymbol{f}}_{i}$, differentials d $\boldsymbol{f}_{i}$ and rule-based priors $\hat{\boldsymbol{f}}_{i}$ using

$$
\begin{aligned}
& \tilde{\boldsymbol{f}}_{1}{ }^{n+1}=\phi_{1} \hat{\boldsymbol{f}}_{1}(\boldsymbol{x})+\left(1-\phi_{1}\right) \sum_{\boldsymbol{y} \in \partial A}\left(\tilde{\boldsymbol{f}}_{1}{ }^{n}+c_{12}\langle\boldsymbol{v}\rangle \tilde{\boldsymbol{f}}_{2}{ }^{n}+c_{13}\langle\boldsymbol{v}\rangle \tilde{\boldsymbol{f}}_{3}{ }^{n}\right) \\
& \tilde{\boldsymbol{f}}_{3}{ }^{n+1}=\phi_{3}\left(\hat{\boldsymbol{f}}_{3}-\left(\hat{\boldsymbol{f}}_{3} \cdot \tilde{\boldsymbol{f}}_{1}{ }^{n}\right) \tilde{\boldsymbol{f}}_{1}{ }^{n}\right)+\left(1-\phi_{3}\right) \tilde{\boldsymbol{f}}_{3}{ }^{n}(\boldsymbol{x}), \quad \tilde{\boldsymbol{f}}_{2}{ }^{n+1}=\tilde{\boldsymbol{f}}_{3}{ }^{n+1} \times \tilde{\boldsymbol{f}}_{1}{ }^{n+1} .
\end{aligned}
$$

Here, $\boldsymbol{v}=\boldsymbol{x}-\boldsymbol{y}, \partial \Omega(\boldsymbol{x})$ denotes the current (diffused) boundary around $\boldsymbol{x}$ from which data is inpainted, and $\phi_{1}=0.1$ and $\phi_{3}=0.7$ are prior weights determined empirically. The higher the confidence in the rule-based model, the larger these coefficients should be. Each $\tilde{\boldsymbol{f}}_{i}{ }^{n+1}$ is normalized after each diffusion pass.

\subsection{Experiments}

Damaged diffusion volumes were simulated using Poisson disk stochastic sampling, where each sample point $\boldsymbol{p}$ satisfies a minimum distance constraint to others. At $\boldsymbol{p}$, an ellipsoid with random semi-axis lengths (range $=1$ to 10 voxels) is carved out. A prototypical synthetic in vivo mask was also obtained by regularly slicing $H$ along its long-axis. We applied these corruptions to a dMRI volume of a healthy rat heart from [12]. We compared our frame inpainting method against a standard vector interpolation scheme based on spatial distance weighting, against a pure vector diffusion scheme using (16) with $c_{i j k}=0$, 
and against a ruled-based model similar to [2] and described in Sec. 6.1. We also tested robustness to noise by combining Poisson sampling (10\% sparsity) and random angular perturbations to $\boldsymbol{f}_{1}$, prior to reconstruction. Table 1 shows the reconstruction of cardiac fibers in a short axis slab near the mid-section from which a chunk of tissue was removed, and from a synthetic transmural cardiac sample obtained using a rule-based helix angle variation (total turning 120 degrees). Our reconstruction method shows a significantly reduced angular error compared to other methods. Table 2 shows Poisson and slicing error maps using our method. Error increases with the amount of damage, and is higher near boundaries. Fig. 4 shows that our frame inpainting outperforms compared methods in minimizing the reconstruction error in all corruption scenarios, and also shows comparable robustness to noise.
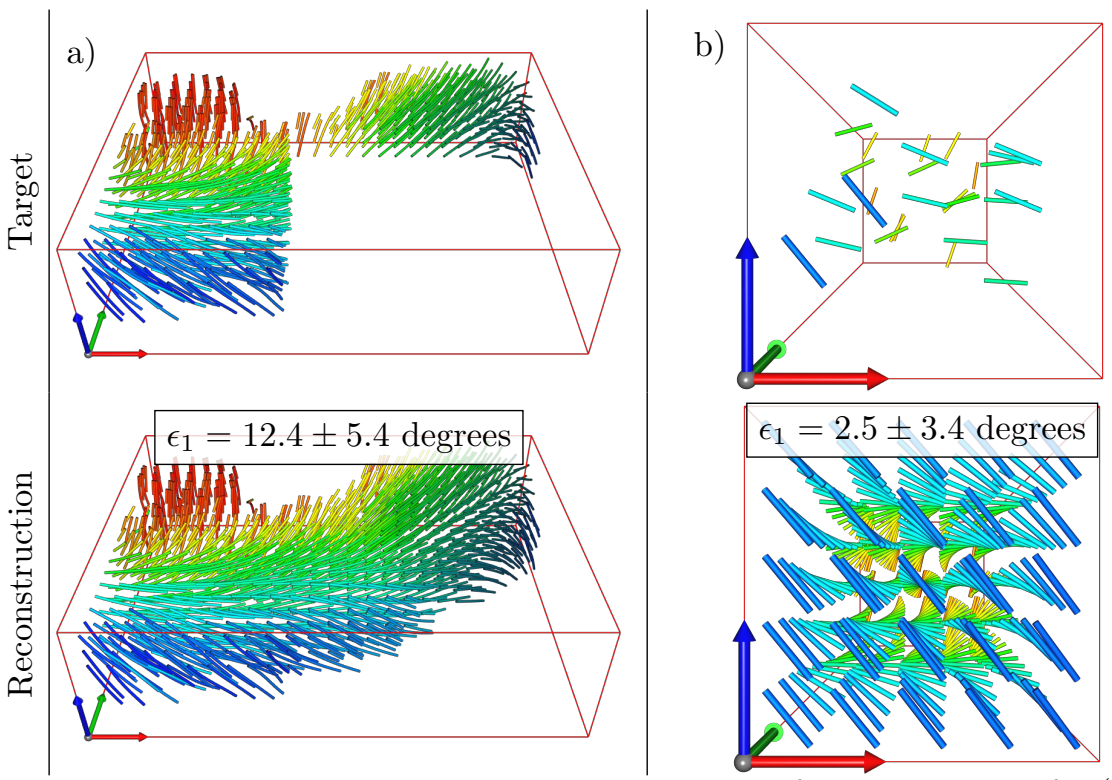

Table 1: Reconstructed volumes and errors using our frame inpainting for (left) a damaged portion of a rat short axis slab, and (right) a sparse (93\% damage) rulebased synthetic field (without rule-based priors). In comparison, vector interpolation yields $(20.6 \pm 10.7,4.5 \pm 5.3)$, vector diffusion yields $(18.6 \pm 9.9,3.7 \pm 3.5)$, and rule-based yields $(14.66 \pm 4.8,-)$ degrees respectively for $\epsilon_{1}(\mathrm{a}, \mathrm{b})$. Colorcoding is based on the helix angle, from -90 (blue) to +90 (red) degrees.

\section{Conclusion}

We proposed a cardiac fiber inpainting method based on moving frame theory, which makes use of a novel closed-form computation for connection forms in 3D frame fields, and incorporates rule-based priors. Our method can fully recover fiber orientations where information is missing, even in very sparse volumes. We 


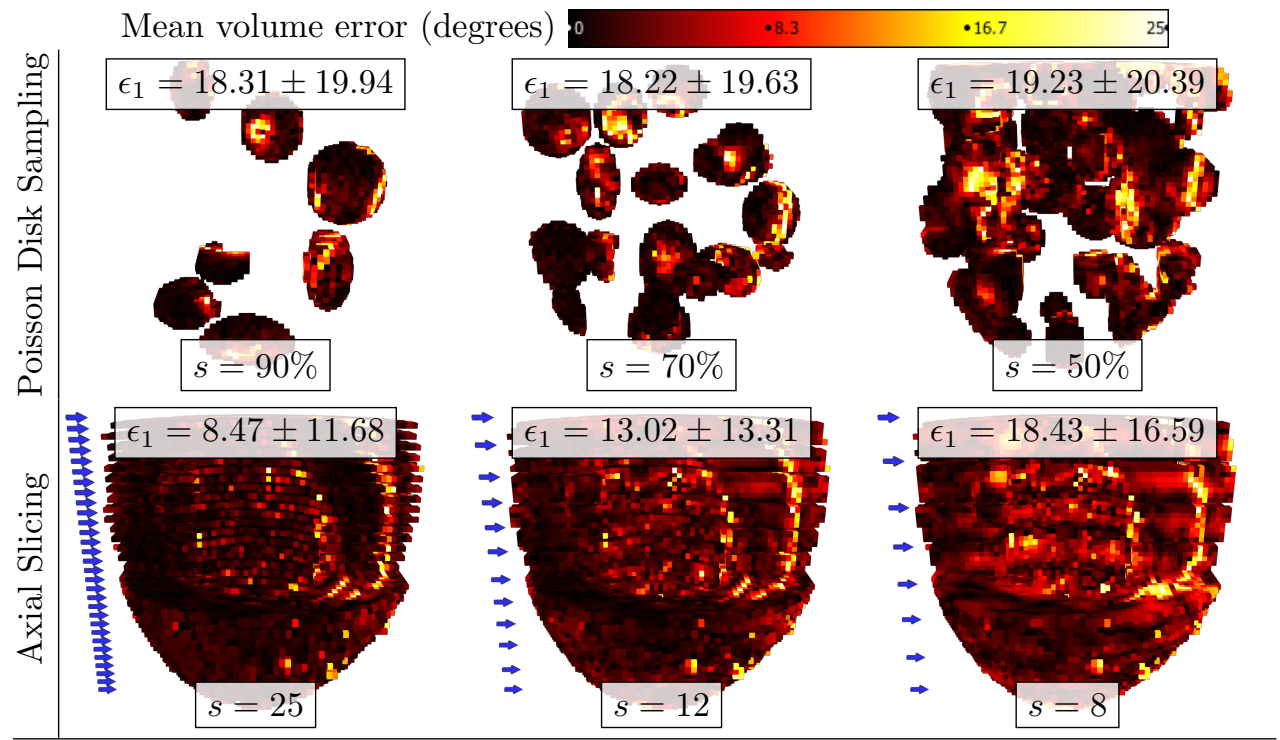

Table 2: (Top) Mean angular reconstruction error $\epsilon_{1}$ between reconstructed fibers and the ground truth (top) for increasingly Poisson-sparse volumes ( $s$ is the percentage of available data) using our frame inpainting methods. 100 realizations were performed, one of which is shown. (Bottom) Reconstruction for increasingly interleaved volumes ( $s$ is the number of slices available), in a long-axis cutout. Available data slices are marked with blue arrows.

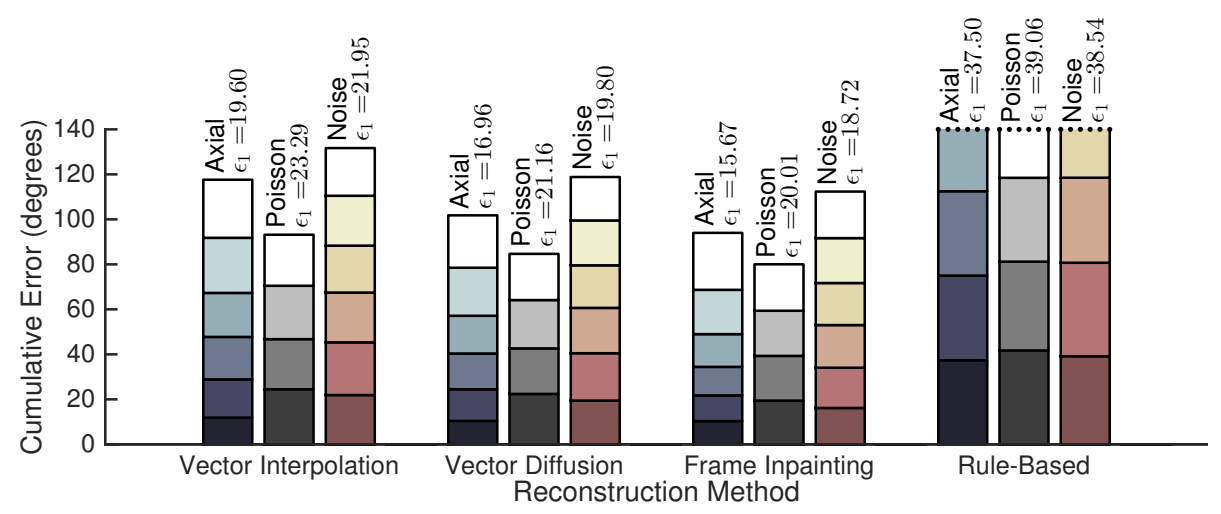

Fig. 4: Mean volume error $\epsilon_{1}$ of reconstruction (degrees) for vector interpolation, vector diffusion, our frame inpainting, and rule-based methods, for various experiment settings: Poisson sampling, axial interleaving, and angular noise. The error is measured as the mean angular difference between reconstructed fibers and the ground truth. Interleaving values increases from 2 to 7 slices; Poisson sampling sparsity ranges from $10 \%$ to $50 \%$; angular perturbations range from $\pm 2^{\circ}$ to $\pm 11^{\circ}$, added to a Poisson-sampled volumes ( sampling and angular noise experiments are averaged out of 100 realizations. 
demonstrated its use by simulating sliced and damaged volumes, and showed that it achieves low error compared to other alternatives, and is robust to noise. We anticipate that the application of these tools could aid computer-assisted guidance and repair of damaged heart walls, and the superresolution of fiber volumes. Future work includes studying higher order and temporal parametrizations of connection forms, the development of better atlas-based cardiac rule-based priors, and patch-based connection form inpainting.

\section{References}

1. Athanasuleas, C.L., Buckberg, G.D., Stanley, A.W., Siler, W., Dor, V., Di Donato, M., Menicanti, L., de Oliveira, S.A., Beyersdorf, F., Kron, I.L., et al.: Surgical ventricular restoration in the treatment of congestive heart failure due to postinfarction ventricular dilation. Journal of the American College of Cardiology 44(7), 1439-1445 (2004)

2. Bayer, J., Blake, R., Plank, G., Trayanova, N.: A novel rule-based algorithm for assigning myocardial fiber orientation to computational heart models. Annals of biomedical engineering 40(10), 2243-2254 (2012)

3. Boutin, M., Bazin, P.L.: Structure from motion: a new look from the point of view of invariant theory. SIAM Journal on Applied Mathematics 64(4), 1156-1174 (2004)

4. Calabi, E., Olver, P.J., Shakiban, C., Tannenbaum, A., Haker, S.: Differential and numerically invariant signature curves applied to object recognition. International Journal of Computer Vision 26(2) (1998)

5. Faugeras, O.: Cartan's moving frame method and its application to the geometry and evolution of curves in the euclidean, affine and projective planes. Springer (1994)

6. Flanders, H.: Differential forms with applications to the physical sciences. Courier Dover Publications (2012)

7. Flash, T., Handzel, A.A.: Affine differential geometry analysis of human arm movements. Biological cybernetics 96(6), 577-601 (2007)

8. Piuze, E., Lombaert, H., Sporring, J., Siddiqi, K.: Cardiac fiber inpainting using cartan forms. In: Medical Image Computing and Computer-Assisted InterventionMICCAI 2013, pp. 509-517. Springer (2013)

9. Piuze, E., Sporring, J., Siddiqi, K.: Moving frames for heart fiber geometry. In: Information Processing in Medical Imaging (2013)

10. Rios, L.M., Sahinidis, N.V.: Derivative-free optimization: A review of algorithms and comparison of software implementations. Journal of Global Optimization 56(3), 1247-1293 (2013)

11. Sapiro, G.: Geometric partial differential equations and image analysis. Cambridge university press (2006)

12. Savadjiev, P., Strijkers, G.J., Bakermans, A.J., Piuze, E., Zucker, S.W., Siddiqi, K.: Heart wall myofibers are arranged in minimal surfaces to optimize organ function. Proc. Natl. Acad. Sci. USA. 109(24), 9248-9253 (2012)

13. Sengupta, P.P., Korinek, J., Belohlavek, M., Narula, J., Vannan, M.A., Jahangir, A., Khandheria, B.K.: Left ventricular structure and function: basic science for cardiac imaging. Journal of the American College of Cardiology 48(10), 1988-2001 (2006)

14. Song, K., Nam, Y.J., Luo, X., Qi, X., Tan, W., Huang, G.N., Acharya, A., Smith, C.L., Tallquist, M.D., Neilson, E.G., et al.: Heart repair by reprogramming nonmyocytes with cardiac transcription factors. Nature 485(7400), 599-604 (2012)

15. Toussaint, N., Sermesant, M., Stoeck, C.T., Kozerke, S., Batchelor, P.G.: In vivo human $3 \mathrm{D}$ cardiac fibre architecture: reconstruction using curvilinear interpolation of diffusion tensor images. In: MICCAI (2010) 